\title{
Light output measurements of the organic light-emitting devices
}

\author{
Yi He, ${ }^{\text {a) }}$ Reiji Hattori, and Jerzy Kanicki \\ Solid-State Electronics Laboratory, Department of Electrical Engineering and Computer Science, \\ The University of Michigan, Ann Arbor, Michigan 48109
}

(Received 13 December 1999; accepted for publication 20 January 2000)

\begin{abstract}
In this article we describe a novel light output measurements method of the organic light-emitting devices (OLEDs). This method not only provides the electroluminescence (EL) spectral response of the OLEDs, but also gives the spectral distribution of the radiant power $(\mathrm{W})$, luminance $\left(\mathrm{cd} / \mathrm{m}^{2}\right)$, luminous flux $(\mathrm{lm})$, and photon emission of the measured light-emitting object. Also an accurate calculation method of the OLED EL external quantum efficiency is proposed. (C) 2000 American Institute of Physics. [S0034-6748(00)02905-1]
\end{abstract}

\section{INTRODUCTION}

Silicon photodiode (or silicon photodiode arrays) and charge-coupled device (CCD) arrays are the most widely used photosensitive detectors for the photoluminescence (PL) and electroluminescence (EL) measurements of the organic light-emitting devices (OLEDs). They feature high sensitivity, stable quantum efficiency over a certain wavelength range, and near-linear response with the incident light power. Both devices are able to simultaneously collect the photosignals over a range of wavelengths. For the OLED brightness (luminance) measurement, in which the EL spectral distribution is not important, the best choice seems to be the calibrated photodiode method. For the external quantum efficiency measurement, a calibrated photodiode could also be used. To determine the absolute PL external quantum efficiency, Greenham et al. developed a method using an integrating sphere and a calibrated photodiode. ${ }^{1}$ However, another work $^{2}$ from the same group using a CCD spectrometer provides several advantages including better accuracy and reliability. To determine the EL external quantum efficiency, most of the research groups still use a calibrated photodiode method. Based on this method, the EL external quantum efficiency can be defined using the following steps: first, the total optical output power $Q$ (measured in watts) is measured using the photodiode; second, the total number of photons emitted is calculated from $Q /$ photon energy; third, the operating current is measured and the total number of electrons is calculated from operating current/e; finally, the external quantum efficiency is calculated from number of photons/ number of electrons. The problem associated with this method is the value of the photon energy used in this calculation. In general, the EL spectrum of the OLED is too broad to be treated as a monochromatic light source, and an average photon energy value has to be used during this calculation. The use of a single photon energy value during this calculation to represent a broad light emission distribution is intrinsically not accurate. Therefore, a systematic error is introduced during this calculation. Moreover, the photon energy value is very difficult to choose when the emission

\footnotetext{
a) Applied Physics Program.
}

spectrum shows multiple peaks or irregular shape. The systematic error further increases when the EL spectrum changes with the external electrical field. ${ }^{3}$ Under this circumstance, the photon energy value has to be recalculated for every single applied voltage. This is not practical for a routine determination of the OLED EL external quantum efficiencies.

In this article we describe a new method to accurately calculate the OLED EL external quantum efficiency using charge-coupled device arrays. A detailed description of the calibration method of the CCD is also provided. Using this method, the wavelength distribution of the OLED radiant power, luminance, and photons emitted can be obtained.

\section{EXPERIMENT}

The CCD spectral analyzer (Instrument S. A.) used in this study consists of a CCD detector, an imaging spectrograph, and an optical fiber. The CCD detector has a 2000 $\times 800$ pixel array and each pixel size is $15 \times 15 \mu \mathrm{m}^{2}$. The optical fiber bundle contains 37 fibers (diameter $200 \mu \mathrm{m} /$ fiber) with an optical response ranging from 200 to $800 \mu \mathrm{m}$. The CCD chip is cooled with the liquid nitrogen during the measurements. The calibration system used in this work is a Labsphere USS-600V Uniform Source System. This system consists of two integrating spheres separated by a motorized, mechanically variable attenuator. A calibration lamp (tungsten halogen) with a known spectral radiance was mounted at the center of the bottom satellite sphere (3 in.). A calibrated luminance meter (photodiode) and an exit port were mounted on the top sphere (6 in.). A baffle was used in the top sphere to prevent light reaching the exit port and the luminance meter directly from the bottom satellite sphere. The experimental setup described above is shown in Fig. 1.

\section{RESULTS AND DISCUSSIONS}

The calibration of a CCD system consists of two steps: first, the CCD response curve is calibrated according to the known spectral radiance of the standard lamp; second, the luminance and photon density distribution of the lightemitting object are derived from the calibrated CCD response curve. 


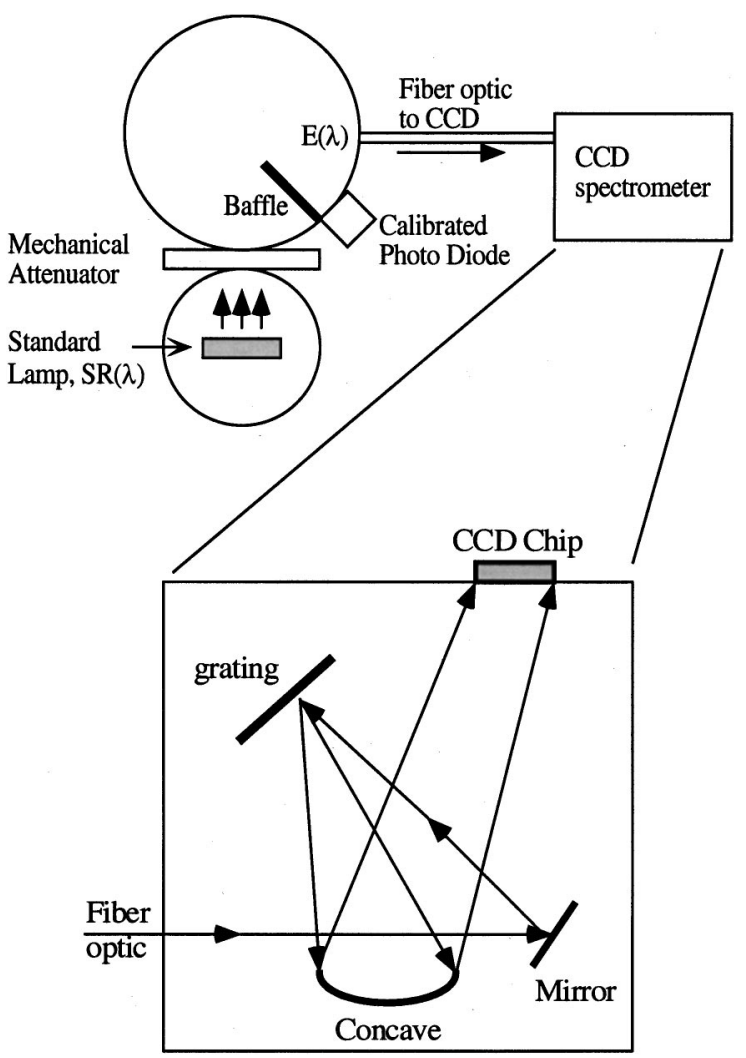

FIG. 1. This diagram illustrates the experimental configuration of the spheres and CCD system used in this calibration. The light path and inside configuration of the CCD spectrometer are also shown.

In the normal conditions, the calibration lamp in the satellite integrating sphere emits a stable spectral radiance which does not change with time. The emitted light passes through the mechanical attenuator and is diffused by the wall of the top sphere. Since the mechanical attenuator is a linear optical element which evenly changes the intensity of the incident light over the wavelengths, the light emission observed at the exit port of the top sphere should always exhibit a same spectrum shape as emitted by the calibration lamp of the bottom sphere. The intensity of the light at the exit port can be changed by varying the attenuator opening. The relationship between the spectral radiance at the exit port and the known spectral radiance of the calibration lamp can then be described by the following equation:

$$
E(\lambda)=A \cdot S R(\lambda),
$$

where $\lambda$ is the wavelength, $E(\lambda)$ is the spectral radiance at the exit port of the sphere, $S R(\lambda)$ is the known spectral radiance curve of the calibration lamp, and $A(\leqslant 1)$ represents the attenuation factor. Due to the nonlinear responses of the optical elements (e.g., the grating) in the CCD system at different wavelengths, the spectral resonance of the CCD system usually does not match the shape of $E(\lambda)$. Therefore, to correctly analyze the spectral distribution curve measured by the CCD system, a correction curve, $\operatorname{corr}(\lambda)$, is needed:

$$
\operatorname{Sp}(\lambda) \cdot \operatorname{corr}(\lambda)=E(\lambda)=A \cdot S R(\lambda),
$$

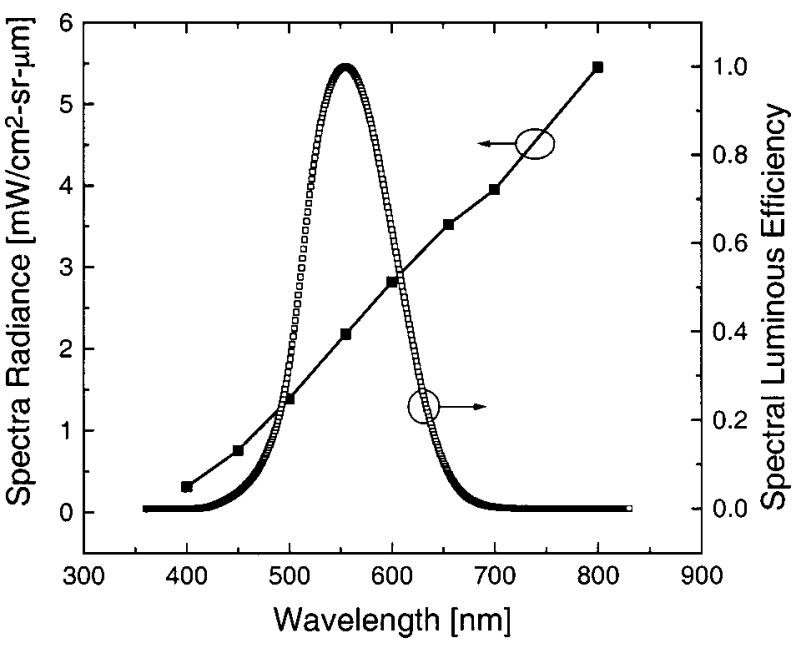

FIG. 2. The spectral radiance of the tungsten calibration lamp in the normal conditions and the normalized CIE photopic spectral luminous efficiency curve of a standard observer.

where $\operatorname{Sp}(\lambda)$ represents the spectral response curve of the CCD. The CCD correction curve, $\operatorname{corr}(\lambda)$, is usually a function of the wavelength and independent of different attenuator openings.

To calculate $\operatorname{corr}(\lambda)$ the radiometric quantity $\left[=A \cdot S R(\lambda), \mathrm{mW} / \mathrm{cm}^{2} \mathrm{sr} \mu \mathrm{m}\right]$ of the light emission at the exit port must be known. However, the luminance meter of the top sphere only outputs a photometric quantity $\left(\mathrm{cd} / \mathrm{m}^{2}\right)$ that is already weighted according to the response of the human eyes. According to photometry theory, the actual photometric quantity obtained from the radiometric emission follows a conversion formula: ${ }^{4}$

$$
\begin{aligned}
\text { Photometric quantity } & =683(\mathrm{~lm} / \mathrm{W}) \int E(\lambda) V(\lambda) d \lambda \\
& =A \cdot 683(\mathrm{~lm} / \mathrm{W}) \int S R(\lambda) V(\lambda) d \lambda,
\end{aligned}
$$

where $V(\lambda)$ represents the CIE photopic spectral luminous efficiency curve for "standard" light adapted eyes. ${ }^{5}$ Therefore, the attenuator factor $A$ can be calculated from photometric quantity/ $\left[683(\mathrm{~lm} / \mathrm{W}) \int S R(\lambda) V(\lambda) d \lambda\right]$. Figure 2 shows the spectral radiance of the calibration lamp and the CIE photopic spectral luminous efficiency curve. Note that only part (400-800 nm) of the original spectral radiance of the calibration lamp was considered during the calculation because the photometric contributions are very small for the wavelength regions below 400 and above $800 \mathrm{~nm}$. For a specific example, the luminance meter reads 4.98 footlamberts $\left(17.06 \mathrm{~cd} / \mathrm{m}^{2}\right)$, the integration $\left[\int S R(\lambda) V(\lambda) d \lambda\right]$ gives $0.242 \mathrm{~mW} / \mathrm{cm}^{2} \mathrm{sr}$, therefore the attenuation factor $A$ $\approx 0.0103$.

Using Eq. (2), the CCD correction curve $\operatorname{corr}(\lambda)$ could be obtained for a specific attenuator opening from the CCD spectral response curve. For the above attenuator setting (luminance of 4.98 foot-lamberts), Fig. 3(a) shows the CCD spectral response curve. The attenuation factor is a function of attenuator setting. However, as discussed previously, the CCD correction curve should remain unchanged for different 


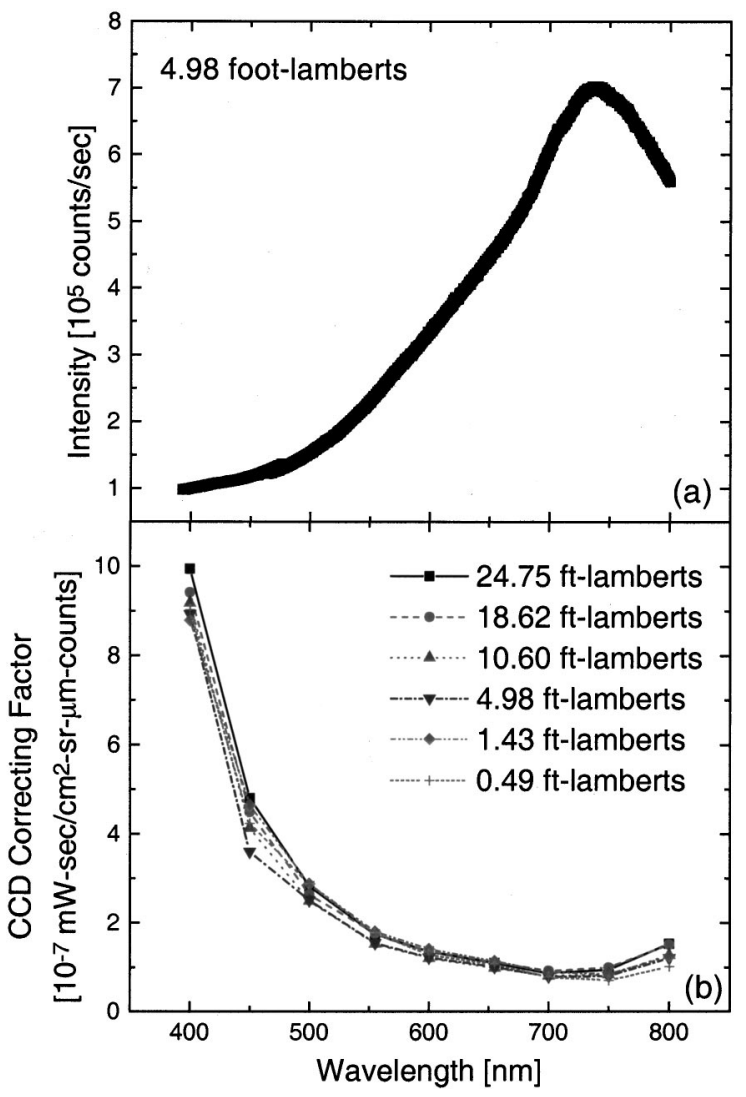

FIG. 3. (a) The CCD spectral response curve for a 4.98 foot-lamberts (17.06 $\mathrm{cd} / \mathrm{m}^{2}$ ) luminance reading. (b) The CCD correction curves for difference attenuator settings.

attenuator settings. This is demonstrated in Fig. 3(b) where several CCD correction curves with different attenuator settings are plotted together. All these CCD correction curves are virtually the same, supporting the arguments made previously.

The actual CCD correction curve is an average of the several CCD correction curves obtained at different attenuator settings. Using this correction curve, the CCD spectral response curve of any visible-range light-emitting object can be converted to its corresponding radiometric spectrum. For the OLEDs, researchers are usually more interested in the brightness (luminance) of the devices than their radiometric spectrum. According to Eq. (2), Eq. (3) can be rewritten as follows:

Photometric quantity $=683(\mathrm{~lm} / \mathrm{W}) \int S p(\lambda) \operatorname{corr}(\lambda) V(\lambda) d \lambda$

$$
=\int \operatorname{Sp}(\lambda) L(\lambda) d \lambda,
$$

where $L(\lambda)=683(\mathrm{~lm} / \mathrm{W}) \operatorname{corr}(\lambda) V(\lambda) . L(\lambda)$ is the conversion function from the CCD spectral response of the lightemitting object to its luminance distribution. Figure 4 shows the CCD luminance calibration curve, $L(\lambda)$. The product of this curve and the $\mathrm{CD}$ spectral response is the luminance distribution of the measured light-emitting object. Because of the influence of the CIE photopic curve $V(\lambda)$, i.e., the human eye sensitivity, the luminance distribution curve usually peaks at around $555 \mathrm{~nm}$ - the same peak location that is

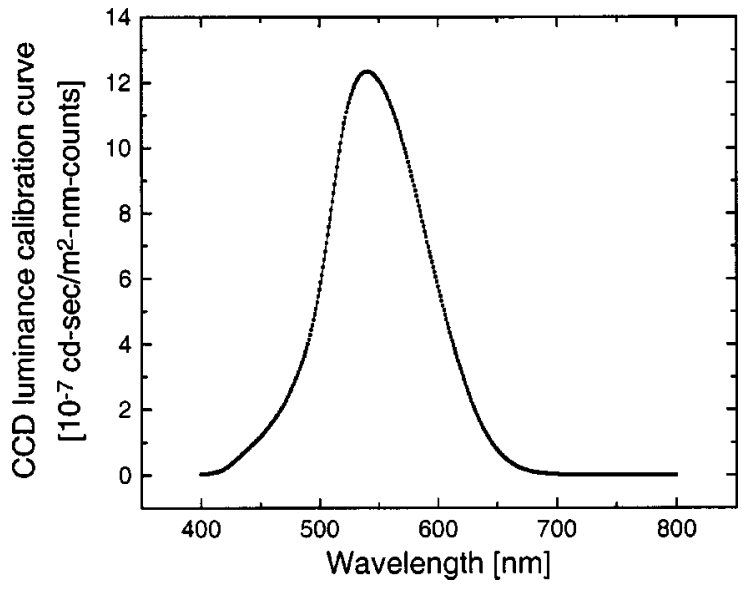

FIG. 4. The CCD luminance calibration curve.

observed in $V(\lambda)$. The actual emission peak location, which should be defined as the most probable emission wavelength, can only be obtained from its photon distribution curve.

Figure 5 illustrates an example of the CCD (previously calibrated with the calibrated light source) EL response curve and the luminance distribution curve of a bilayer OLED. ${ }^{6}$ In this case the OLED, operated at a current density of 0.324 $\mathrm{mA} / \mathrm{cm}^{2}$, has a green light emission. The total luminance (area under the luminance distribution curve in Fig. 5) of this OLED is $\sim 40.83 \mathrm{~cd} / \mathrm{m}^{2}$.

The external quantum efficiency of the OLED represents the number of externally emitted photons per electron produced by the external circuit. In this case the density of the produced electrons is represented by the current density applied to OLED. The photon density, on the other hand, can be calculated from the OLED EL spectrum.

From the spectral radiance [a product of $\operatorname{corr}(\lambda)$ and the CCD response curve] of the OLED, it photon density distribution over the wavelength can be obtained:

$$
p h(\lambda)=\frac{\pi S p(\lambda) \operatorname{corr}(\lambda)}{h v}=\frac{\pi S p(\lambda) \operatorname{corr}(\lambda) \lambda}{h c},
$$

where $h$ is the Planck's constant, $c$ is the velocity of the light,

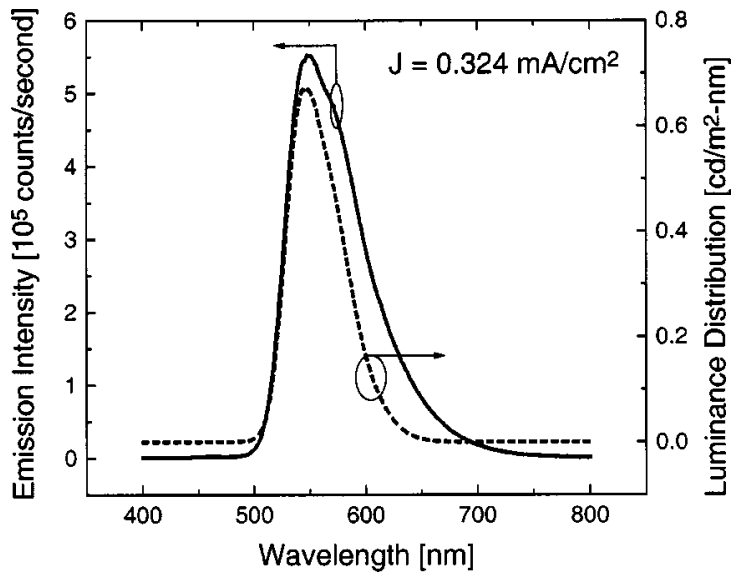

FIG. 5. The CCD spectral response of the OLED operated to 0.324 $\mathrm{mA} / \mathrm{cm}^{2}$, and the corresponding luminance distribution curve. 


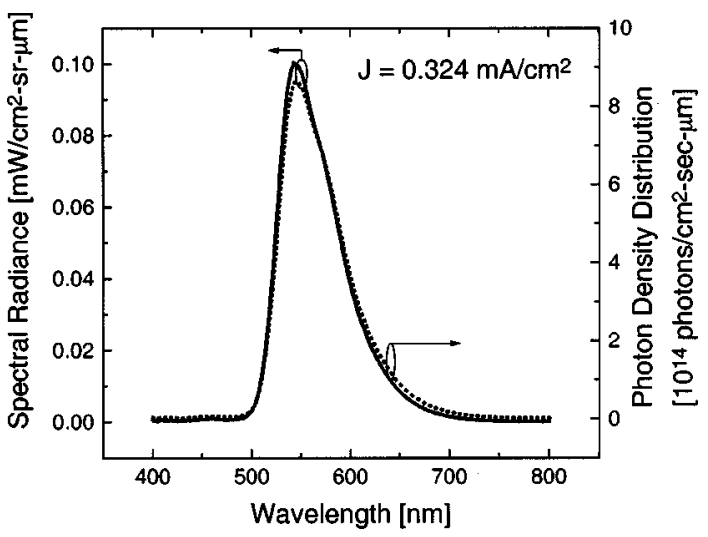

FIG. 6. The spectral radiance of the OLED operated at $0.324 \mathrm{~mA} / \mathrm{cm}^{2}$, and the corresponding photon density distribution curve.

and $p h(\lambda)$ is the photon density distribution. Factor $\pi$ is an integration factor induced by assuming Lambertian emission. $^{7,8}$

Figure 6 illustrates the spectral radiance obtained for a bilayer OLED and its photon density distribution. ${ }^{6}$ According to the photon density distribution, the external quantum efficiency can then be derived from the following equation:

$$
\eta_{e}=\frac{\int p h(\lambda) d \lambda}{J / e},
$$

where $J$ is the current density applied to the OLED. For the example given above, $J$ is $0.324 \mathrm{~mA} / \mathrm{cm}^{2}$, the integration of Fig. 6 yields $6.76 \times 10^{13}$ photons $/ \mathrm{cm}^{2} \mathrm{~s}$, therefore the external quantum efficiency calculated using this method is $3.3 \%$. For this specific case the OLED brightness was $40.83 \mathrm{~cd} / \mathrm{m}^{2}$.

\section{ACKNOWLEDGMENTS}

The authors would like to thank Professor Stephen Rand for valuable discussions. The financial support of this research is provided by the Center for Display Technology and Manufacturing at the University of Michigan, and DARPAONR grant (N0014-99-1-0958).

${ }^{1}$ N. C. Greenham, I. D. W. Samuel, G. R. Hayes, R. T. Phillips, Y. A. R. R. Kessener, S. C. Moratti, A. B. Holmes, and R. H. Friend, Chem. Phys. Lett. 241, 89 (1995)

${ }^{2}$ J. C. de Mello, H. Felix Wittmann, and R. H. Friend, Adv. Mater. 9, 230 (1997).

${ }^{3}$ M. Berggren, O. Inganas, G. Gustafsson, J. Rasmusson, M. R. Andersson, T. Hjertberg, and O. Wennerstrom, Nature (London) 372, 444 (1994).

${ }^{4}$ T. N. Cornsweet, Visual Perception (Academic, New York, 1970).

${ }^{5}$ L. Levi, CRC Handbook of Tables of Functions for Applied Optics (Chemical Rubber, Cleveland, OH, 1974).

${ }^{6}$ Y. He, S. Gong, R. Hattori, and J. Kanicki, Appl. Phys. Lett. 74, 2265 (1999).

${ }^{7}$ N. C. Greenham, R. H. Friend, and D. D. C. Bradley, Adv. Mater. 6, 491 (1994).

${ }^{8}$ G. Gu, D. Z. Garbuzov, P. E. Burrows, S. Venkatesh, and S. R. Forest, Opt. Lett. 22, 396 (1997). 